\title{
FRANÇOISE COLLIN, IL LAVORO DEL PENSIERO
}

\author{
MARA MONTANARO
}

\section{Doctoranda de la Università del Salento i de l’Université Descartes (Paris 5)}

\begin{abstract}
Si intende in questo testo ricostruire brevemente il percorso intellettuale di Françoise Collin, tra letteratura, filosofia e femminismo. Mettendo in luce la sua assoluta originalità e la coerenza strutturale e non dialettica che accompagna tale percorso, la nozione di praxis può essere letta come la categoria attraverso la quale si sviluppa tale percorso. Praxis della scrittura (Blanchot), praxis nella vita politica (Arendt), praxis nel femminismo (rapporto teoria/azione).
\end{abstract}

PALORE CHIAVE: Femminismo, frammentario, praxis, rivoluzione permanente, dialogo.

\section{Françoise Collin, el trabajo del pensamiento}

Este texto intentará reconstruir brevemente el recorrido intelectual de Françoise Collin entre literatura, filosofía y feminismo. A partir de su absoluta originalidad y la coherencia estructural y no dialéctica que acompaña tal recorrido, la noción de praxis puede ser leída como la categoría por medio de la cual éste se desarrolla: praxis de la escritura (Blanchot), praxis en la vida política (Arendt), praxis en el feminismo, relación teoría/acción.

PALABRAS ClAVE: Feminismo, pensamiento, praxis, revolución permanente, diálogo.

\section{Françoise Collin, the labor of thought}

The aim of this text is to briefly reconstruct Françoise Collin's intellectual trajectory in literature, philosophy, and feminism. The notion of praxis can be read as the category that guides her work, and it draws attention to her originality as well as the structural, nondialectical coherence of her thought: praxis of writing (Blanchot), praxis in the political (Arendt), praxis in feminism, the relationship between theory and action.

KEY WORDS: Feminism, thought, permanent revolution, dialogue.

\section{Die welt ist fort, ich muss dich tragen. \\ Paul Celan}

La vita è stata quello che è stata, quello che poteva essere, tra scrittura, filosofia e politica. La vita non è uno sviluppo logico, c'è un impeto dell'esistenza che, tra coerenza e incoerenza, eccede ogni specie di giustificazione a priori e anche a posteriori.

F. C. 
Vorrei dunque qui con voi tracciare l'itinerario di Françoise Collin, un percorso atipico e particolare tra scrittura, letteratura e filosofia che si annoda nel contesto del movimento femminista degli anni Settanta (dopo una prima e breve esperienza politica che è quella del comunismo), ma che si sviluppa anzitutto a partire dal 1971 in Belgio e più precisamente a Bruxelles dove fonda e anima allora la prima rivista femminista in lingua francese, i Cahiers $d u$ Grif, in rapporto costante con la Francia, ma lontano dai dibattiti e dalle querelle ideologiche e personali che scuotono il movimento parigino.

Il primo numero dei Cahiers du Grif appare in ottobre del 1973, l'11 novembre dello stesso anno, giorno della seconda giornata delle donne a Bruxelles, sono vendute 1500 copie ed è esaurito la sera stessa e contemporaneamente è distribuito a Parigi alla libreria Maspero. Rapidamente, la rivista assume una dimensione internazionale nel mondo francofono. L'editoriale del primo numero che annuncia altresì il ritmo con cui i Cahiers saranno pubblicati, interroga il giovane movimento femminista ponendo la questione "Le féminisme pour quoi faire?".

Françoise Collin incontra Jacqueline Aubenas che manifesta il suo interesse per il progetto dei Cahiers, un po' prima dell'11 novembre 1972. In concreto i Cahiers nascono a casa di Françoise Collin, il primo numero è pubblicato con fondi propri, i Cahiers si collocano al margine dell'istituzione, procedono con la volontà di costruire insieme, come dice Collin, fuori dal circuito degli uomini senza attendere che loro autorizzino la pubblicazione nelle loro riviste.

Riflessione dunque tra donne, condividere la scrittura con altre donne, essi sono il prodotto della riflessione e del lavoro di una ventina di collaboratrici regolari intorno alle quali si aggiungono numerose altre collaboratrici. Il gruppo è non misto e si vuole pluralista, composto di femministe storiche e della seconda vague. È dunque composto di donne intellettuali ma anche da lavoratrici, donne immigrate, casalinghe.

I Cahiers, opera collettiva, sono un supporto all'azione militante, le partecipanti che provengono, come abbiamo detto, da orizzonti diversi sono dunque inscritte nel femminismo in modo differente.

Questa diversità è presente fin anche nella composizione e nel modo di redazione dei Cahiers: i temi nascono dalla preoccupazione delle partecipanti e il contenuto della pubblicazione è il risultato di una discussione che vuole rendere accessibile a tutte la scrittura e le parole delle donne. Ogni partecipante è invitata a esprimersi a partire dalla propria sensibilità e esperienza, poco conta il suo sapere o le sue competenze in materia. Oltre a questa volontà di liberazione della parola, i Cahiers $d u$ Grif perseguono un lavoro che supera la semplice condivisione delle soggettività. Ogni tematica proposta è un campo da esplorare al servizio del femminismo, all'interno del quale teoria e prassi sono mescolate. Al di fuori dell'editoriale, ogni articolo è firmato da una o più autrici, contrariamente a quanto faceva allora il MLF in Francia. Per i primi numeri, il 
testo rende conto delle interazioni ed è annotato dalle differenti lettrici al margine, che occupa quasi la metà della pagina, tale pratica è assolutamente originale e soprattutto unica se si pensa all'ingiunzione del MLF alla fusione e all'abolizione della singolarità nell'anonimato. Queste note sono altresì delle referenze bibliografiche e testimoniano l'intenso lavoro di riflessione collettiva e dialettica. Le riunioni preparatorie sono registrate e decriptate dopo dal comitato di redazione, l'esperienza messa in forma di racconto, testimonianza, serve sia da materiale bruto alla pubblicazione sia da tappa per la concettualizzazione.

Inviteranno d'altronde le teoriche delle differenti correnti del femminismo francese a presentare le loro tesi specifiche, ma la prima intenzione dei Cahiers mira a procedere in qualche modo al dubbio metodico nei confronti del sapere istituito, contaminato dalla dominazione maschile, per lasciare spazio alle testimonianze delle donne di tutte le condizioni e di tutte le origini che si presentano alle riunioni che organizzano nei quartieri, testimonianze che sono giustapposte ai testi di intellettuali o scrittrici che sollecitano.

È negli Stati Uniti, dopo un viaggio nel 1970, che Collin percepisce la vitalità del movimento femminista, erede del movimento hippie, movimento non tanto intellettuale quanto esistenziale e culturale: gruppi di autocoscienza, gruppi di self-help, gallerie d'arte, case delle donne, giornali, riviste. È come un nuovo modo di vivere che è inventato e che illumina le sue erranze new-yorkesi.

Il suo impegno attivo nel movimento femminista nascente viene ad interrompere un percorso intellettuale e letterario estremamente significativo. Aveva, in effetti, già pubblicato due romanzi (Collin, 1960; 1962) nella collezione di Jean Cayrol nelle edizioni Seuil dove la rivista Tel Quel diretta da Philiph Sollers iniziava a muovere i suoi passi e nelle edizioni Gallimard pubblica Maurice Blanchot et la question de l'écriture il primo studio critico completo consacratogli in Francia che tutt'oggi resta una delle monografie di riferimento su Blanchot.

Questo impegno è stato senza dubbio favorito se non determinato dalla misoginia del milieu filosofico della sua università che era esclusivamente maschile e poco disposto a far spazio a una donna.

Nel 1978 termina la prima serie dei Cahiers. É il momento dei bilanci, di una riflessione sull'azione fatta in questi cinque anni. Il Cahier riunisce un insieme di testi critici e testimonianze. Françoise Collin fonda con Hedwige Peemans l'Université des femmes che durerà due anni; nel 1981 si trasferisce a Parigi, nel momento della svolta che incarna la presidenza socialista di François Mitterand, svolta che va poco a poco ad articolare l'insurrezione iniziale del femminismo all'istituzione tanto politica che universitaria e che darà luogo agli studi di genere.

In questa congiuntura transitoria che tiene insieme ancora margine e istituzione, i Cahiers $d u$ Grif, pubblicati ora nelle edizioni Tierce che dirige Françoise Pasquier, conosceranno una seconda fase, differente, ma feconda 
quanto la prima, fase ormai più legata al milieu intellettuale parigino ma sempre nella preoccupazione di un pensiero plurale che non si trasforma in ideologia e che si lascia investire dalle domande.

Nello stesso tempo apre e dirige una collezione di opere di donne prima nelle edizioni Minuit, la collezione Grif, dopo nelle edizioni Tierce, la collezione Littérales, dove a partire dal 1985, pubblica un numero importante di traduzioni di Hannah Arendt alla quale inizia a interessarsi agli inizi degli anni Ottanta e sulla quale co-organizza un convegno al Collège International de Philosophie nel 1981. Era il primo convegno consacrato ad Arendt che introduceva in Francia la lettura gauchiste della filosofa tedesca.

Le donne filosofe riconosciute sono rare, ma Collin riconosce in Arendt una concezione del mondo plurale, dove ciascuno/a si manifesta attraverso la parola e attraverso l'azione a partire dalla sua propria situazione. Si tratta di uno dei nodi che più ispirerà l'opera di Collin che ad Arendt dedicherà un numero dei Cahiers du Grif, il 33 nel 1986.

Due altri numeri dei Cahiers $d u$ Grif assicurano l'articolazione tra filosofia e femminismo: uno Provenances de la pensée, vede la collaborazione di donne filosofe che non si sono tuttavia dichiarate femministe ma che rispondono tutte all'invito di Collin. L'altro, che sarà l'ultimo, è consacrato a Sarah Kofman. (Collin ha organizzato dopo la sua morte, con la filosofa Françoise Proust, un convegno di omaggio, convegno al quale anche Derrida, vi partecipa). Il numero sarà tradotto in giapponese.

Nel 1990, avendo perduto la sua infrastruttura editoriale, Collin pone fine alla rivista. I tempi sono cambiati, l'epoca del margine è passata: il femminismo si è trasformato in studi di genere e istituzionalizzato.

Delle riviste universitarie e anche disciplinari si sostituiscono poco a poco alle riviste di riflessione generale dell'epoca femminista. (Segnalo che tutti i numeri della rivista sono ora disponibili sul sito Persee grazie all'Università di Paris Descartes in collaborazione con l'Università di Lione).

Il lavoro del pensiero di Françoise Collin continua a inscriversi puntualmente in contesti universitari e militanti in Francia come nel resto del mondo dove è invitata a fare conferenze o corsi, sempre tra i due registri: quello del pensiero e quello dell'azione.

In occasione di un invito a occupare la Chaire Franqui all'Università di Liegi in Belgio nel 2006, assicura teoricamente l'articolazione dei differenti aspetti e dei differenti autori che hanno segnato la sua opera: Maurice Blanchot, Emmanuel Levinas, Hannah Arendt.

Appartiene, Françoise Collin, alla generazione delle prime femministe che hanno tenuto insieme pensiero e azione, lavoro individuale e lavoro collettivo, fuori dall'istituzione e spesso senza infrastrutture e non alla generazione degli studi di genere. 
In effetti, Collin non è nata con il femminismo né dal femminismo come alcune delle sue contemporanee, la sua avventura politica e intellettuale non è cominciata con il movimento.

Il femminismo ha rappresentato per lei piuttosto una sorta di conversione, parola che utilizza la stessa Collin, che ha interrotto e fatto deviare un percorso letterario e filosofico e ne ha sconvolto profondamente il corso.

Il femminismo è dunque stato per lei una rottura e, nello stesso tempo, l'inizio di un'avventura collettiva.

\section{La politica: dal marxismo al femminismo}

Il femminismo non è stato tuttavia il primo terreno politico che l'ha sollecitata o che ha sollecitato la sua generazione.

In effetti, tra la fine della seconda guerra mondiale e il maggio '68, la figura salvifica della politica si è presentata anzitutto sotto la forma del marxismo, incarnato dai regimi comunisti che volevano realizzare una società senza classi.

Dottrina seducente, rassicurante anche, e che Collin qualifica come un surrogato della religione, poiché prometteva l'accesso al paradiso in terra attraverso un rimedio radicale: il superamento della relazione capitale/lavoro, proponeva un modello sociale ed umano risolutore, sostiene Collin, facendo così l'economia del duro lavoro di ricerca congiunturale.

Sottolinea Collin che il materialismo dialettico, la praxis marxista, è paradossalmente una teoria prima di essere un regime politico; mentre il femminismo è un movimento, una pratica prima di elaborare le sue teorizzazioni se si fa eccezione al riferimento più mitico che teorico a Il Secondo Sesso.

Emergendo dalla guerra e dai suoi traumatismi, i giovani della sua generazione, sostiene Collin che all'epoca aveva vent'anni, non potevano che essere sedotti dalla promessa del paradiso in terra. L'ideologia di un sistema supposto perfetto rendeva vano ma anche pericoloso ogni tentativo di pensare a partire da se stessi (Arendt) paralizzando o anche interdicendo ogni iniziativa singolare. $\hat{E}$ in quel momento che sente l'irriducibilità del pensiero come pensiero critico a qualsiasi sistema.

Qualche anno dopo il maggio '68, attraverso il femminismo sottopone dunque la sua riflessione e la sua azione ad un campo politico completamente differente: un campo dove il collettivo nasceva o anche sollecitava al contrario le singolarità, un campo dove la teoria, se di teoria si può parlare, si formulava punto per punto nella congiuntura dell'esperienza stessa.

Il femminismo è stato all'origine un vero materialismo, sostiene Collin, poiché non risultava dall'applicazione di una teoria prestabilita ma doveva inventarsi passo dopo passo, richiedendo l'apporto di ciascuna e dove il gruppo non poteva pensarsi senza l'intervento di ciascuna: l'immaginazione al potere o ancora il pensiero in atto, eccedendo ogni ideologia. 
Il Secondo Sesso di Simone De Beauvoir non ha, in effetti, avuto per lo sviluppo del femminismo lo stesso statuto dogmatico rigido del Il Capitale per il marxismo.

$\grave{E}$ un riferimento simbolico, più che dogmatico, nonostante il successo della formula lapidaria che funziona come un simbolo o uno slogan: donna non si nasce, si diventa. Pochi, in effetti, hanno all'epoca analizzato nel dettaglio e ricollocato nell'insieme dell'opera e della vita, ciò che avviene oggi dopo quasi cinquant'anni dopo, la complessità polisemica dell'enunciato e le contraddizioni interne dell'opera proprio perché Il Secondo Sesso di Simone de Beauvoir è stato a lungo per molti una referenza, un luogo d'ispirazione, più che una dottrina. È un personaggio mitico e un'opera mitica. Considerata con distacco, sostiene Collin, l'opera appare d'altronde molto più polisemica e complessa e non può racchiudere la formula feticista isolata dal suo contesto.

Così nel femminismo la dottrina non ha preceduto l'azione: pensare e agire si sono costantemente coniugati, l'uno chiamava l'altro. Un vero materialismo, a conti fatti, poiché il pensiero si costituiva poco a poco, attraverso forme diverse nel crogiolo e nella contingenza delle esperienze e anche dei problemi che la congiuntura impone.

$\grave{E}$ in senso proprio un movimento, uno spazio di interpretazione permanente dove teoria e azione si concatenano, rinviano l'uno all'altro, seguono il loro corso in maniera autonoma, una provocazione a essere e a pensare piuttosto che un dogma.

Il femminismo, sostiene Collin, è una rivoluzione nella durata, un'insurrezione permanente, non riposa sulla rappresentazione della sua fine, né su una dottrina prestabilita, si pensa e s'inventa ad ogni passo, pertanto deve mantenersi come movimento, come azione che si formula e riformula. Tale rivoluzione, che Collin ama definire permanente, presenta una specificità rispetto alla rivoluzione marxista: da una parte è una rivoluzione non evenemenziale o violenta, ma è nella durata, dall'altra non riposa sulla rappresentazione della sua fine né su una dottrina prestabilita ma si pensa e s'inventa ad ogni passo. C’è nel marxismo una pretesa scientifica volta alla determinazione, sostiene Collin, di un modello speculativamente elaborato; il femminismo non soccombe a questo rischio nella misura in cui è innanzitutto definito come un movimento: il movimento di liberazione delle donne, come un'azione che formula e riformula le sue problematiche, ma non c'è una rappresentazione a priori né di una società ideale né dei cammini da percorrere per crearla.

Il femminismo ha instaurato una politica non metafisica, vale a dire una politica che non riposa su una rappresentazione definita né dei suoi obiettivi né dei suoi metodi ed è una politica dell'azione permanente, è materialista fino in fondo.

Con le parole di Françoise Collin: 
Le véritable abandon de tout postulat métaphysique (ou naturaliste) des sexes, c'est le dépassement de la position unitaire autant que duelle. J'ai parlé de l' "irreprésentable" mais aussi de la "praxis" des sexes. Les questions doivent toujours être portées, elles ne doivent pas nous mettre à la question sous la forme d'une alternative. [...] J'ai toujours voulu laisser réinterroger la pensée par la pratique et accompagner la pratique par la vigilance de la pensée. Je recours volontiers au concept de praxis, rencontré dans le marxisme, mais présent aussi chez Arendt dans sa référence à Aristote, pour désigner à la fois la pensée et la pratique. Arendt pense que le politique ne peut être commandé par une philosophie préalable qui lui fournirait sa norme, mais que le politique comme agir suscite constamment son propre objet. L'imagination et la faculté de juger sont pour elles les qualités essentielles du politique. La pensée est une vigilance qui accompagne et qui éclaire, mais non une théorie qui détermine l'action à partir d'une représentation préalable des objectifs auxquels le réel aurait à se plier. [...] il est vrai que le caractère passionnant du féminisme était, et reste pour moi, lié à ce projet premier: dessiner le paysage de la liberté plutôt que d'en définir les objets. L'insurrection ou si on préfère la déconstruction que l'institution. (Rochefort et Haase-Dubosc, 2001: 4)

Il movimento femminista è dunque un movimento rivoluzionario che non si appoggia su alcun modello prestabilito e che non può pensarsi in termini di riappropriazione o ritorno. Poiché l'oppressione dei sessi è originaria, la sua risoluzione non può essere pensata che in termini di avvenire. L'essere delle donne come l'obiettivo del femminismo è dell'ordine dell'irrappresentabile e la politica femminista è una politica del desiderio e non del bisogno, politica dell'immaginazione e non applicazione di un sistema.

\section{L'esperienza americana}

È dopo un soggiorno negli Stati Uniti, come sottolineavo inizialmente, che Collin fa l'esperienza concreta del femminismo come movimento politico e sociale già presente, articolando il singolare e il collettivo senza un'armatura filosofica o ideologica prestabilita, nonostante un'ammirazione e dei commentari precoci di Simone De Beauvoir.

Senza aver affrontato né risolto i dibattiti distruttivi sullo statuto della differenza dei sessi, tra universalismo e differenzialismo, che lacerano la scena francese, le donne si riuniscono in collettivi con obiettivi concreti e i gruppi di autocoscienza vi articolano la singolarità propria di ciascuna alla costituzione di un collettivo senza presupporre un'ideologia comune. La pratica precede in qualche modo la teoria. È questa pratica che non attende di aver affinato la sua 
teoria che la seduce, vi ritrova una forma di materialismo o almeno di pragmatismo: agire prima di sapere, agire per sapere.

\section{Il dubbio metodico dopo Descartes}

Questo empirismo americano non la porta ad elaborare o a mettere a punto la giusta teoria dei sessi ma a fare: creare qualcosa di collettivo con le donne e anzitutto riunire alcune donne intorno a questo progetto: i Cahiers du Grif. Questo progetto intellettuale e concreto, dei Cahiers, nasce dunque a Bruxelles nel 1973, prima rivista femminista in lingua francese, rivista che conoscerà un grande successo sia in Francia sia in altri paesi. La rivista deve avere per oggetto, nelle intenzioni di Collin, non tanto la costituzione di una dottrina comune, ma anzitutto la costituzione di un essere insieme delle donne, suscitando l'iniziativa di ciascuna in un dialogo plurale (espressione arendtiana che scopre più tardi). Da vera materialista o nello spirito del maggio ' 68 Collin ritiene che la pratica preceda la teoria e la generi o almeno l'accompagni.

Si tratta, in effetti, di ripartire da zero ("Partisans année zero" è il titolo del primo numero della celebre rivista femminista) a partire da un nuovo dubbio metodico che potrebbe riferirsi a quello di Descartes, dubbio metodico che si concentra sul sapere pervertito da secoli di fallocrazia (come i marxisti avevano denunciato la scienza borghese).

A differenza delle riviste che sono nate dopo a Parigi, come ad esempio Questions Féministes, nessuna teoria (almeno cosciente ed esplicita) precede il testo né la selezione dei testi pubblicati. Inspirata piuttosto dall'empirismo americano la rivista si propone di far nascere e di sostenere delle iniziative, di mettere in rilievo delle testimonianze e delle ipotesi, piuttosto che di elaborare una dottrina. L'anno zero assomiglia un po' al dubbio metodico e alla tabula rasa di Descartes: una certa diffidenza nei confronti della teoria, sempre normativa e inficiata di un sapere fallocratico a profitto dell'espressione spontanea e plurale delle donne.

Da qui l'importanza nella rivista delle testimonianze a fianco di articoli più teorici, priorità di una pratica anche disparata sulla teoria e del dialogo sul discorso, la verità non si identifica al sapere ma piuttosto al dialogo.

In un secondo tempo, la riflessione rifluisce verso la sua vocazione teorica e si situa poco a poco in riferimento alle ricerche che sono state progressivamente elaborate e pubblicate. La questione politica delle donne necessita allora dello studio critico della fondazione e del funzionamento della democrazia, in particolare nella sua forma repubblicana francese che è stata al centro di tutti i dibattiti.

Questa dislocazione d'attenzione e del contesto di riflessione si riferisce anche ad una dislocazione dell'itinerario soggettivo della stessa Collin. È nel 1981 in effetti che, come abbiamo detto, lasciando il Belgio dove aveva vissuto i primi 
dieci anni di impegno e di riflessione femminista, attraverso la fondazione dei Cahiers du Grif e dell'Université des femmes, si trasferisce a Parigi.

In effetti, se le idee e le rivendicazioni sono state comuni alle donne ai due lati della frontiera e degli scambi numerosi tra le femministe belghe e le femministe francesi hanno avuto luogo dall'inizio in modo che l'insieme della scena è familiare, il contesto storico e istituzionale della Francia è specifico e questa specificità si accentua a partire dal momento in cui il femminismo abbandona il suo carattere di movimento autonomo per dialogare sempre di più con l'istituzione.

$\grave{E}$ dunque in questo contesto che la riflessione di Collin va e si confronta con il femminismo francese, le questioni determinanti del rapporto del movimento all'istituzione si impongono allora in un confronto storico: come e perché l'instaurazione democratica e repubblicana ha ignorato le donne? La democrazia nella forma che ha preso da due secoli è suscettibile di far spazio alle donne? Non è piuttosto una struttura incapace di far spazio alle donne senza costringerle a divenire "uomini come gli altri"?

La necessità del confronto teorico e pratico con le istituzioni occulta l'importanza della trasformazione dello spazio simbolico che non può essere identificata con la trasformazione delle sfere della rappresentazione politica.

Dunque correlativamente all'analisi delle forme dell'istituzione con la quale le donne sono ormai condotte, l'attenzione di Collin si sofferma parallelamente sulle forme del linguaggio e dell'arte che costituiscono in qualche modo un potenziale dell'incondizionalità nel momento in cui la politica é sotto condizione. Quest'attenzione ha sempre caratterizzato l'opera di Collin, i suoi primi scritti sono dei romanzi e il primo lavoro filosofico la conduce, attraverso l'opera di Blanchot, alla questione della scrittura. Quest' attenzione iniziale è significativa nella misura in cui è legata ad un rapporto originario alle parole piuttosto che all'imperativo del discorso teorico e più ancora al rischio dell'ideologia politica.

In quest'itinerario riflessivo che si sviluppa parallelamente all'analisi dell'opera arendtiana si impone, per Collin, l'idea che la concezione stessa della modernità democratica comporta una resistenza ad una dimensione inseparabile dell'esperienza delle donne: quella della generazione.

\section{Effetti sul suo itinerario singolare}

Questa conversione al femminismo in qualche modo selvaggia scuoteva la sua immagine di scrittrice e di filosofa, quest'immagine e questa reputazione che le avevano assicurato la pubblicazione dei romanzi e del saggio su Blanchot. È stata violentemente attaccata durante dei convegni filosofici e letterari nei quali fino ad allora era invitata prima di essere sempre più scartata o tenuta a distanza con prudenza e per anni ha lavorato a distanza dall'Università. 
La sua stigmatizzazione dal milieu filosofico doveva essere lunga e lasciare delle tracce ma Collin ritrova credito a partire dalla scoperta dell'opera di Arendt, in una libreria americana agli inizi degli anni Ottanta.

Come sappiamo, Arendt non era femminista, ma poneva fondamentalmente la questione del mondo comune, dell'essere insieme, fondando la pluralità sui differenti. Arendt metteva il dialogo nel cuore dell'essere insieme, cioè della politica, e chiariva filosoficamente una serie di questioni che Collin si poneva.

La lettura e gli studi arendtiani suggellano la difficile conciliazione o riconciliazione dell'impegno politico femminista e della riflessione filosofica di Collin che ad Arendt dedica un numero considerevole di articoli prima di dedicarle un volume più sintetico (Collin, 1999).

Una buona parte del suo lavoro successivo, a partire dagli anni Ottanta, è consistito nell'assicurare teoricamente e politicamente la compatibilità del suo lavoro filosofico e della sua attività femminista.

Gli studi di genere universitari che si sono sviluppati in tutte le discipline in Francia dopo gli Stati Uniti possono apparire come il terreno di una tale riconciliazione, di una tale conciliazione per la nuova generazione, ma la loro problematica è articolata da un punto di vista scientifico, in qualche modo in maniera immutabile.

Ciò che interessa Collin riguardo al pensiero è la molteplicità dei suoi punti di vista, la dislocazione coerente, ma non unificata dei suoi approcci.

\section{Genesi intellettuale}

La prima parte dell'itinerario filosofico di Collin precede dunque, come abbiamo visto, l'emergenza del femminismo e a fortiori quella degli studi di genere, ed è legata all'avventura della scrittura letteraria. La scrittura e la pubblicazione dei due romanzi e dei racconti e la pubblicazione con Gallimard del saggio filosofico di Blanchot, pensatore e scrittore in quegli anni stimato solo da una cerchia ristretta di intellettuali e che non era mai stato oggetto di uno studio pongono Collin tra gli intellettuali più all'avanguardia.

Blanchot sarà più tardi rivendicato se non appropriato da Derrida, ma Collin nella redazione del suo libro aveva già identificato e segnalato ciò che le sembrava una vicinanza intellettuale tra i due.

$\grave{E}$ in ogni caso nell'esperienza letteraria e della scrittura che Collin apprende definitivamente che la verità non è riducibile a una tesi né a un concetto ma che essa si dispiega in una frase infinita, che la verità si scandisce infinitamente e si dispiega ma non ci si può appropriare di essa. La teoria, per Collin, è un relais che domanda presto di essere superata dal pensiero: pensare eccede la teoria che fissa e rassicura. Ella aveva da tempo rinunciato a credere che la storia collettiva o singolare si sviluppi secondo una linea continua, dialettica o no ma non ha mai rinunciato ad una forma di coerenza nell'incoerenza stessa, coerenza attraversata 
dalla molteplicità, dalla moltitudine e che trova la sua forza in questa stessa demoltiplicazione: un Uno strutturale piuttosto che dialettico, abitato dal principio della ripetizione più che da quello del progresso.

Nell'opera di Collin il concetto di praxis introduce e sostiene la coerenza incoerente di un itinerario di più di quarant'anni di riflessione e di scrittura, è un concetto che getta una certa luce nuova sulle dimensioni apparentemente disparate di un percorso, sia nei suoi momenti singolari come nei suoi momenti collettivi, è infatti la sola a parlare di praxis della differenza e ad articolare tale concetto a quello di différend dei sessi nella misura in cui la differenza dei sessi è un agire, una praxis appunto, senza rappresentazione prestabilita, un atto, l'atto di disfare le false evidenze.

Si tratta di un agire, un far essere senza rappresentazione della sua fine e senza postulato di una qualsiasi totalità potenziale. È infatti nel senso arendtiano che Collin legge la praxis, l'agire, opposto alla fabbricazione, alla poeisis.

E importante vedere come Collin ha voluto che Arendt, una donna il cui pensiero è stato per lei enormemente importante, sia stata riconosciuta come fonte e risorsa di pensiero, non come risorsa del pensiero femminista, ma come risorsa di pensiero tout court.

Quando iniziò a studiare Arendt, veniva fuori da un periodo lungo consacrato al militantismo: l'opera arendtiana le permetteva di ricreare un legame con la sua vita filosofica. Non era per lei un oggetto di studio solamente ma uno spazio di ritorno al pensiero, era la messa in valore di una donna filosofa la cui opera diviene un motivo di ispirazione per i filosofi, uomini e donne.

Inizia a tradurre le sue opere con Tierce nella collezione che le era stata affidata, traduzioni che sono state riprese senza domandare il suo assenso nelle edizioni Puf e il primo convegno, come dicevamo sopra, al Collège International de Philosophie nel 1981.

Così Arendt non è solamente appropriata internazionalmente dalle femministe intellettuali, ma diventa ugualmente un riferimento della scena filosofica in quanto tale: per gli uomini filosofi è una donna filosofa ma che fortunatamente non è femminista; per le donne è una donna che non è femminista ma che ha preso un posto importante in filosofia.

Ciò che interessa Collin, in Arendt, è soprattutto la sua concezione dell'agire plurale, il compito permanente di far essere il mondo senza alcun modello. Distingue, in effetti, la fabbricazione (poiesis) come esecuzione di un modello e l'azione (praxis) che è un'avventura, che apre delle piste senza garanzia preliminare e che è il luogo della politica; politica che è una posta in gioco che dipende in permanenza da tutti coloro che vi partecipano e non è l'applicazione di una dottrina preliminare. Come sostiene altresì Diane Lamoreux in Pensées rebelles. Autour de Rosa Luxemburg, Hannah Arendt et Françoise Collin, ella non si interessa alle riflessioni di Hannah Arendt sui paria, anche se sarebbe stato interessante applicare alle donne l'analisi che quest'ultima sviluppa a proposito 
degli ebrei. Ciò che le interessa in Arendt è la sua concezione della politica, concezione che la porta a considerare differentemente il rapporto del femminismo alla politica e soprattutto le nozioni di agire come praxis, di pluralità e natalità.

Con le parole di Françoise:

La rencontre de son oeuvre en 1985 a marqué mon retour à la philosophie, après les années d'un féminisme privilégiant le terrain. Sa réflexion sur le politique, et sur les rapports du politique et du privé, me permettait au moins de mettre de l'ordre dans une expérience un peu chaotique, de prendre distance avec elle pour l'assumer. [...] Deux articulations m'ont semblé éclairantes concernant la définition du politique : pluralité et natalité. Pluralité du monde commun, le commun n'étant pas menacé mais constitué au contraire non seulement par la pluralité, mais par les différences et les divergences, qu'assume le dialogue. Natalité, c'est-à-dire rapport continuel à ce qui commence, assomption de la temporalité comme innovation (Collin avec Kaufer, 2005: 162)

Pensare, come agire, non è elaborare una teoria inglobante, ma giudicare e decidere in ogni congiuntura. Il senso richiede la vigilanza e l'iniziativa costante, il pensiero non può ridursi ad una teoria sulla quale fondarsi una volta per tutte. Collin trova in Arendt un privilegio permanente del pensiero e del giudizio sulla fissità della teoria.

Contemporaneamente introduce l'opera arendtiana nella sfera della riflessione femminista, non presentandola certo come la norma o la referenza assoluta, ma come colei che le ha permesso in un momento del suo itinerario di ricreare un legame con la filosofia senza occultare la posta in gioco femminista che aveva segnato il suo lavoro dagli inizi degli anni Settanta.

Tale legame tra l'aspetto più propriamente politico femminista del suo percorso e l'aspetto più filosofico trova spazio, come abbiamo detto prima, nei Cahiers du Grif attraverso alcuni numeri: nel 1986 quello consacrato a Hannah Arendt o quello in Provenances de la pensée dove riunisce una serie di donne filosofe che si occupano di temi diversi, senza doversi definire come femministe né occuparsi del genere; alla morte di Sarah Kofman, pubblicherà gli atti del convegno organizzato con la collaborazione della filosofa Françoise Proust.

Noi donne, sostiene Collin, siamo certo le titolari della questione del genere, ma noi siamo anche al di là di questa questione: noi siamo ormai titolari di tutta l'estensione del pensiero. Gli studi di genere sono un trampolino, non devono essere una trappola. Cosi Arendt, per Collin, è al di qua del nostro lavoro nella misura in cui non accede alla questione del genere, ma al di qua nella misura in cui affronta sovranamente l'estensione intera del campo filosofico etico o 
politico. Così come ha affermato che bisogna essere femminista, ma bisogna essere più che femminista, Collin sostiene altresì che bisogna passare per gli studi di genere, ma bisogna andare al di là poiché essi possono diventare un confinamento a loro volta.

Bisogna dunque pensare nel quadro degli studi di genere ma anche al di là. $\mathrm{E}$ un'ingiunzione pericolosa e delicata alla quale dobbiamo rispondere, questo difficile punto di equilibrio, questa posizione transdisciplinare è la posta in gioco del pensiero e della ricerca oggi: introdurre il parametro del genere nella ricerca senza farne un parametro esclusivo o causale.

Si tratta, piuttosto, di rispondere al dato, alla congiuntura, di agire senza rappresentazione di un fine ideale, anche se le tentazioni ideologiche e dogmatiche sono ricorrenti. La tentazione delle parole che salvano c'è sempre, ma l'agire politico non è una dottrina della salvezza, della buona forma, è un movimento. Pensare e agire si accompagnano reciprocamente: pensare e agire e agire pensando. La rappresentazione del migliore dei mondi possibili è solo una tentazione ricorrente e sempre presente poiché sostituisce all'agire avventuroso una rappresentazione salvifica.

Un movimento invece, tale è il modo in cui Françoise Collin ha vissuto il femminismo e ne ha fatto esperienza nella scrittura, restando fedele alla nozione di praxis che spesso ha rivendicato nel suo significato non marxista ma aristotelico.

$\mathrm{Si}$ tratta dell'intreccio tra teoria e pratica, un intreccio che lascia all'elaborazione teorica la sua parte attiva: pensare un fenomeno non è solamente renderne conto è anche farlo essere, scrivere non riflette il reale ma lo costituisce.

Il femminismo come politica, per Collin, ma anche come pensiero, è un movimento permanente, un pensiero in atto e non una teoria fissata: fa essere e nello stesso tempo rende conto. Pensare così come agire, non è solamente riflettere il reale ma contribuire a costituirlo: c'è un potere delle parole anche se come gli atti, le azioni, le parole possono essere dei feticci morti, degli strumenti che hanno perso la loro forza creativa. Dire è fare, come diceva Austin e il pensiero e la parola non sono un riflesso del reale ma sono un'azione e contribuiscono all'azione. Le nostre parole anche se non producono la rivoluzione, dislocano il senso, sostiene Collin, e questo è il nostro lavoro. Infinito. Scrivere, pensare, agire trovano così nell'esperienza di una vita non la loro sintesi ma almeno la loro relazione.

Nell'avventura del femminismo, Collin è rimasta fedele al doppio registro che è simbolizzato dai suoi autori privilegiati: Blanchot, da una parte, con il registro della poetica, della scrittura letteraria ma anche dell'arte, e Arendt dall'altra parte, che guida la sua riflessione nel registro della politica. L'hanno protetta entrambi dalle derive dogmatiche che minacciano ogni impegno politico, le hanno insegnato sia l'uno che l'altra, afferma Collin, che non esiste un mondo ideale, ma che la mobilizzazione permanente di ognuno è necessaria 
perché ci sia mondo comune. Tra la poetica e la politica, che sono i suoi due registri maggiori, si disegna tuttavia la questione etica.

Tale questione etica ha interrogato il suo percorso politico a partire da due prospettive: quella del dialogo, così come si impone nella lettura dell'opera di Levinas, amico di Blanchot e che ha conosciuto come tale, e quella della preoccupazione costante dell'alterità.

In effetti, se il movimento femminista ha saputo costituire un legame politico tra donne, legame assente prima poiché esistevano una per una, la dimensione etica è stata a lungo sottostimata. Ora non c'è un mondo comune plurale per parlare con le parole di Arendt che non necessiti del dialogo e del rispetto dell'altro nel disaccordo come nell'accordo.

Il femminismo del XX secolo è stato dunque un'avventura incerta della sua fine e dei suoi mezzi, una scommessa sull'avvenire. È diventato dopo, l'abbiamo voluto, un oggetto identificabile, universitario e politico. Questi proponimenti condivisi tra l'elogio dell'avventura politica collettiva e l'elogio della creazione sempre singolare possono sembrare contraddittori, sostenere questa contraddizione piuttosto che tentare di risolverla in qualche falsa sintesi è l'imperativo di un'esistenza, dell'esistenza di Françoise Collin che ha sempre sostenuto il dialogo plurale, in sé e con le altre, con i nostri contemporanei, con chi ci ha preceduto e con chi ci seguirà.

Il dialogo infinito ne è stato l'istanza. Il movimento femminista mira alla realizzazione delle condizioni di possibilità d'espressione delle donne, in generale, nel mondo che le ha secolarmente paralizzata ma nello stesso tempo mira al superamento di questa generalità in vista dell'affermazione di singolarità irriducibili.

È là tutta la sua ambiguità e il suo rischio: il passaggio attraverso il comune può permettere l'affermazione di ciascuno/a e nella pluralità dialogale trovare la sua unità.

Movimento dunque in cui ciascuna deve trovare il suo posto originale, quello di un'articolazione indispensabile tra l'esigenza singolare e il rapporto al comune, tra l'assunzione di ciò che è e di ciò che deve essere. Il movimento di liberazione delle donne è dunque animato da un principio di libertà, piuttosto che d'uguaglianza.

L'uguaglianza, se consiste in un uguagliamento di tutti secondo un modello imposto dallo Stato non è la democrazia ed essa viene dal basso, scrive Collin, dalla società civile, l'uguaglianza non è nulla senza libertà. La democrazia comincia da noi stesse e in noi stesse, non è per un'idea della donna che noi lottiamo, ma per la libertà delle donne a manifestarsi attraverso la parola e l'azione come scriveva Arendt.

Il femminismo è, per Collin, la ricerca delle condizioni che permettono a ciascuno e a ciascuna di vivere la propria vita. Non c'è democrazia, sostiene 
Collin, laddove le donne non sono co-decisionali del mondo comune, esse non devono dunque essere solamente integrate nel progetto della società definita senza di loro, ma devono poter partecipare alla sua definizione a tutti i livelli. Bisogna dunque lavorare nello stesso tempo su e nelle istituzioni per utilizzarle e trasformarle ma anche fuori dalle istituzioni tradizionali e identificare i principali nodi costitutivi dell'oppressione: corpo, creazione, linguaggio, lavoro, maternità, sessualità, ecc.

Il termine più adatto a descrivere il percorso di Collin tra scrittura, femminismo e filosofia è forse quello di cheminement, un camminare nello stesso tempo fatto di continuità e di dislocamenti e liberato a un certo polimorfismo.

Un camminare verso un'identità, ma un'identità composita, camminare che è d'altronde quello di un pensiero che non si immobilizza in una teoria definita una volta per tutte ma che è che è chiamato a interrogarsi senza sosta. La teoria, per Collin, costituisce un quadro fisso al quale attenersi per valutare le nuove questioni, non è legata al momento dell'interrogazione ma dell'affermazione: determina i contorni di una posizione, isola le cause, seduce per la sua chiarezza rassicurante, ma rischia sempre di trasformarsi in ideologia.

Il pensiero interroga, segue il suo cammino, nutre e strappa nello stesso tempo la teoria e il movimento delle donne è un andare verso.

Il femminismo è, per Collin, un lavoro di reinvenzione delle posizioni sessuate e del mondo comune, è un andare verso ciò che non c'è ancora, nel linguaggio come nell'agire, far essere il nuovo, anche se non è una rivoluzione ma una dislocazione. Ha sempre voluto lasciare reinterrogare il pensiero attraverso la pratica e accompagnare la pratica attraverso la vigilanza del pensiero. Una praxis dunque il femminismo, dove il pensiero è una vigilanza che accompagna e chiarisce, ma non una teoria che determina l'azione a partire da una rappresentazione prestabilita degli obiettivi ai quali il reale dovrebbe piegarsi.

L'essenza della politica consiste nell'elaborazione e nella messa in forma del terreno d'azione, non nella scelta tra due oggetti predeterminati o nell'ingiunzione del per o contro. Il carattere appassionante del femminismo è e resta per Collin legato al primo progetto: disegnare il paesaggio della libertà piuttosto che definirne gli oggetti, l'insurrezione o se si preferisce la decostruzione piuttosto che l'istituzione.

Femminismo come politica dunque dell'irrappresentabile e come spiega in un'intervista Collin stessa, l'irrappresentabile corrisponde profondamente alla sua concezione del carattere avventuroso della scrittura, del pensiero e dell'azione. Non si ha una rappresentazione a priori di ciò à cui miriamo, anche politicamente, di quella che sarebbe «la società giusta».

Il femminismo è una politica dell'irrappresentabile, un agire che richiede l'immaginazione piuttosto che un fabbricare determinato da un modello. La vita politica, la vita tout court, come la scrittura è un'assunzione di rischi, un andare verso, senza rappresentazione della sua fine che l'unità apparente del libro 
compiuto, la fine della storia, rileva e nasconde allo stesso tempo. La scrittura così come la vita è un mouvement permanent sans que le mot fin soit jamais prononcé attraverso «un agir trasformateur conjoncturel, ici et maintenant» (Duverger, 2012).

\section{TESTI CITATI}

Collin, Françoise (1960), Le jour fabuleux, Paris, Seuil.

-(1962) Rose qui peut, Paris, Seuil.

-(1999), L'Homme est-il devenu superflu? Hannah Arendt, Paris, Edile Jacob. avec Irène Kaufer (2005), Parcours féministe, Bruxelles, Éditions Labor.

Duverger, Sylvie, "Françoise Collin ou la non-clôture en partage", Le nouvel observateur [en ligne] 05/09/2012.

http://feministesentousgenres.blogs.nouvelobs.com

Rochefort, Florence et Danielle Haase-Dubosc, "Entretien avec Françoise Collin. Philosophe et intellectuelle feminist", CLIO. Histoire, femmes et sociétés [en ligne], 13/2001. 19/06/2006, http://clio.revues.org/1545; DOI : 10.4000/clio. 1545 